\title{
Handover Management based on Asymmetric Nature of LTE-A HetNet using MCDM Algorithm
}

\author{
Xianda Chen \\ College of Information \& Communication Engineering, Sungkyunkwan University, \\ Suwon, 440-746, Korea \\ E-mail: chenxianda@skku.edu \\ Young Ho Suh, Seung Wan Kim, and Hee Yong Youn \\ College of Information \& Communication Engineering, Sungkyunkwan University, \\ Suwon, 440-746, Korea \\ E-mail: $\{y$ hseo, secret77,youn7147\}@skku.edu
}

\begin{abstract}
This paper proposes an efficient handover scheme considering the asymmetric characteristics of macro and pico cells in LTE-A HetNet. The proposed scheme detects a suitable target eNB for handover using a multi-criteria decision making algorithm and the mobility information of user equipment (UE). A prompt handover is triggered for the UE if the received power is too low or the downlink SINR is below a threshold, avoiding radio link failure. The simulation results reveal that the proposed scheme significantly enhances goodput while reducing packet loss and unnecessary handover.
\end{abstract}

Keywords: Handover, MCDM, LTE-A, HetNet, Goodput.

\section{Introduction}

Conforming to the forecast from the fields [1], the global mobile data traffic has been exponentially growing in recent years. The demands on mobile data are driven by smartphones, tablets, laptops and other portable devices, and the plain cellular networks are insufficient to provide the services with limited radio resources. Besides increasing the capacity of networks, thus, the data rate of crowded regions as well as cell edges needs to be increased where the performance can be significantly degraded. Heterogeneous networks
(HetNets), tightly integrated low-power nodes (LPNs) such as pico-cells and femto-cells inside large-scale macro-cells, can improve the network performance by offloading from large macro-cells of hot-spot coverage. They can also provide high quality service at macro-cell edges where the signal coverage significantly degrades. As illustrated in Fig. 1, a two-tier macro-pico LTE-A HetNet consists of three main components: evolvedNodeB (eNB), Mobility Management Entity (MME), and Serving Gateway (S-GW)/Packet Data Network Gateway $(\mathrm{P}-\mathrm{GW})$. The eNB, including macro eNB (MeNB) and pico eNB (PeNB), performs the radio 
control functions such as packet scheduling and handover. MME is in charge of authorization, bearer establishment, and roaming. S-GW forwards data from and to eNB to serve user equipment (UE), while P-GW provides UE with the access to exterior network.

Dense deployment of small cells in HetNets introduces numerous challenges such as mobility management, downlink-uplink asymmetry, cell association, interference management, etc [2]. Among these challenges, mobility management is of great significance due to the dropped calls after handover when UEs move across the coverage of LPNs. Handover hysteresis (Hyst) and time-to-trigger (TTT) window are typically used to make appropriate handover decision in LTE environment. Here, handover is triggered only when Event A3 condition is satisfied during the TTT window. It is reasonable to handover low-mobility UE, for example a pedestrian, to an LPN. However, handover may be unnecessary for highmobility UE when it quickly passes through the coverage of an LPN, avoiding the resource-consuming ping-pong effect. Moreover, high-mobility UE may move deep into the coverage of an LPN before the TTT expires, incurring late handover or even handover failure [3].

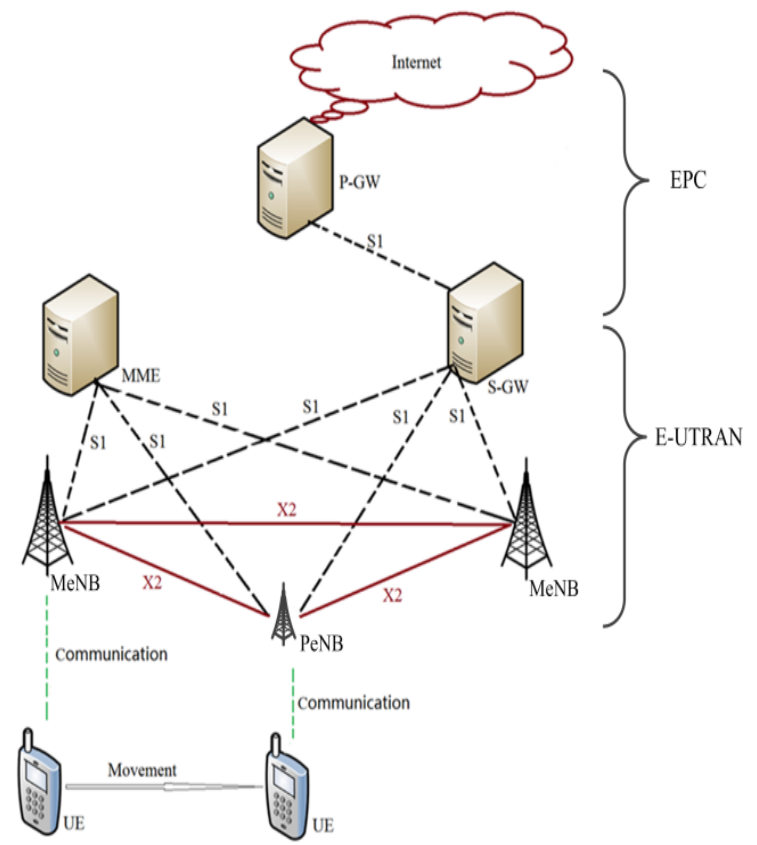

Fig. 1. The structure of LTE-A network.

The improvement on the mobility of HetNet can be achieved with cell range expansion (CRE) [4] and enhanced inter-cell interference coordination (eICIC)
[5] including almost blank subframe (ABS). Mobility state estimation (MSE) [6] and UE-speed-based method [7] can also be the solution. An autonomous mobility state estimator for UE was presented in [8], trying to minimize the number of handover of high-speed UEs on Pico cells. A number of researches focused on the selfoptimization of handover parameters for improving the mobility in HetNet. The TTT parameter is adjusted based on the type of handover in [9]. The scheme in [10] adjusts the handover parameters by global optimization, and then local optimization of cellspecific parameters is applied. [11] suggested to adjust the cell-pair handover parameters based on the type of handover failures and the ping-pong rate. [12] introduced the concepts of dwell probability and handover priority to update the handover parameters.

Because of the asymmetric property of macro-cell and LPN, however, the strategy treating all handover scenarios equally as with the conventional homogeneous network is not suitable for HetNet. The proposed scheme thus considers two special scenarios in HetNet environment. First, even though the received signal from the serving cell of a UE is high enough for communication, the handover preparation step is triggered for the UE any time when its downlink signal to interference plus noise ratio (SINR) drops below a threshold to avoid radio link failure (RLF). Second, when the received power is too low, if the UE detects a suitable target eNB of good quality, the handover is also triggered promptly even before the TTT window expires to avoid RLF. The proposed scheme selects a suitable target eNB for handover when the UE moves. To provide the service of high quality to the UE, a novel handover mechanism considering the complexity of the two-tire HetNet (macro-pico) environment is proposed in this paper. The proposed scheme adopts the MultiCriteria Decision Making (MCDM) algorithm to rank the neighbor eNBs, and the handover decision is then made as the UE moves in and out the pico-cell. The simulation results reveal that the proposed scheme greatly reduces the number of unnecessary handovers and packet loss rate, and increases the goodput compared to the representative existing schemes.

The rest of the paper is organized as follows: Section 2 provides the related work. Section 3 presents the proposed scheme, followed by simulation and performance evaluation in Section 4. Finally, we conclude the paper in Section 5. 


\section{Related Work}

In this section the handover procedure of LTE is presented first, and then the basic handover scheme used in LTE is briefly described.

\subsection{The Procedure of Handover}

The 3GPP LTE handover procedure typically consists of four phases: measurement phase, preparation phase, execution phase, and completion phase. Figure 2 briefly describes the handover procedure in LTE-A. Handover measurements are usually based on the estimation of downlink reference signal received power (RSRP) in Layer_1 (physical layer), while the effects of fading and estimation imperfection are filtered out in Layer_3 (network layer) [13]. When the filtered measurements satisfy a certain condition for handover, the UE sends a Measurement Report (MR) to the serving eNodeB so that it can initiate the handover preparation phase. Based on the $M R$ and radio resource management (RRM), the serving eNodeB starts to negotiate with the neighbor eNBs and selects the best target eNB to which the UE is handed over. This operation is called handover decision.

The serving eNB sends a Handover Request to the target eNodeB, which contains all the relevant handover information. The target eNB then responds with a Handover Request Ack message, providing the information on the establishment of a new radio link the target eNB grants. The handover process comes into execution phase when the UE receives "handover command" message from the serving eNB; meanwhile, the serving eNB forwards all packets of the UE to the target eNB. The UE sends "handover confirm" message when it successfully accesses the target eNodeB. The completion phase also includes switching the downlink path from the serving eNB to the target eNB and releasing radio resources associated to the UE from the serving eNB.

\subsection{The Decision of Handover}

There are eight types of conditions for handover entry specified in 3GPP: event A1, A2, A3, A4 and A5 for intra-Radio Access Technologies (RAT) inter-carrier handover; event A6 for intra-RAT intra-carrier handover; event $\mathrm{B} 1$ and $\mathrm{B} 2$ for inter-RAT handover [13]. Event $A 3$ is common for triggering handovers in LTE, where the L3 RSRP of the target eNB is greater than that of the serving eNB. Once event A3 occurs, the UE starts the TTT timer. Hyst indicates the value of a handover margin between the serving eNB and the target eNB. It identifies the most appropriate target eNB the UE should be handed over to. The TTT_timer is used for reducing ping-pong handovers. The serving eNB decides to trigger handover if the condition of event A3 is met for the entire duration of TTT.

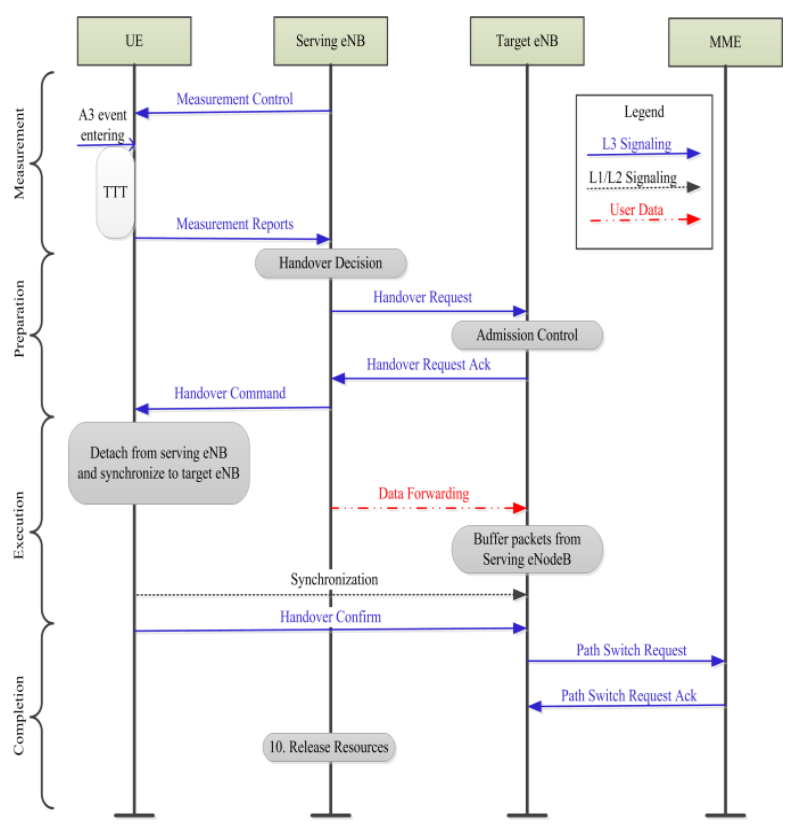

Fig. 2. The handover procedure specified in LTE Release 10.

\section{The Proposed Scheme}

Dense deployment of LPNs inside macro cells causes the structure of the LTE network to be complicated. The received signal power of a UE varies during its movement depending on the type of eNB from which it gets service. Redundant neighbor eNBs around the serving eNB dramatically degrades the downlink SINR of a UE. Therefore, the basic handover scheme will not be efficient in LTE, where the timing of handover is controlled by pre-defined value of Hyst and TTT.

\subsection{Destination of Handover}

In eNB-rich HetNet environment, making handover decision based on only the received RSRP may degrade the performance of service since the eNBs located near the serving eNB may influence the quality of the signal. Besides, the residence time of a UE in an eNB is of great importance to avoid triggering handover into the 
cell from where the UE will move out soon. Hanover due to in and out continuously between two cells, called ping-pong effect, significantly wastes signaling resources. The UE on the edge of the serving cell may also locate in the overlapped area of the radio range of other neighbor eNBs because of the dense deployment of LPNs. Then a wrong target eNB might be chosen for handover, resulting in RLF. Therefore, it is crucial to conspicuously decide the target eNB when handover is triggered for a UE.

The proposed scheme employs a MCDM method, Technique for Order of Preference by Similarity to Ideal Solution (TOPSIS) [14], to rank the preferences of the neighbor eNBs. Here four criteria are considered: the movement direction of the UE, the residence time in the target cell, the interference condition of the received signal, and the power of the received signal from the serving eNB.

As shown in Figure 2, the moving direction of a UE can be modeled as the angle of $\angle P_{e} P_{t-1} P_{t}, \beta$, where $P_{t}$ is the current position of the UE at time $t, P_{t-1}$ is its previous position, and $P_{e}$ is the position of the target eNB, respectively. $\beta$ is calculated by Eq. (1) along with the path of the movement of the UE. The less the value of $\beta$, the better handover the UE gets. This is because the UE receives strong signal when it moves towards the target eNB with a small $\beta$.

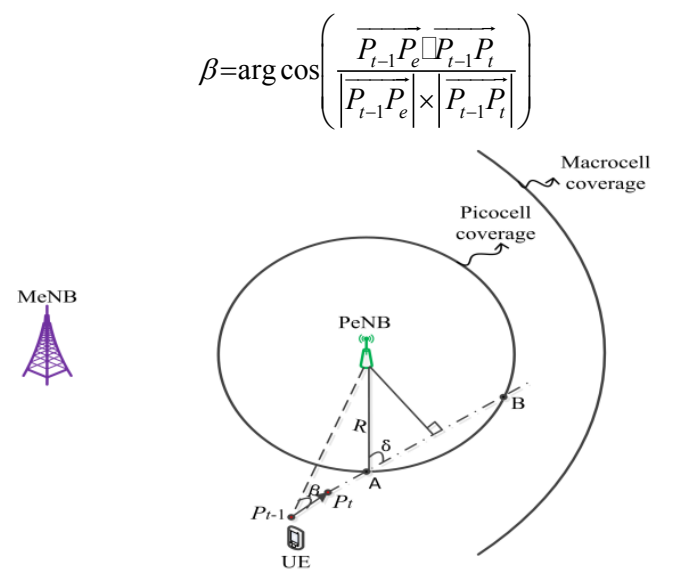

Fig. 2. The measurement of the movement direction of UE.

Assuming that the UE moves straight across the coverage of the target eNB, the residence time, $T_{R}$, can be obtained by Eq. (2), where $R$ is the radius of the signal coverage. $T_{R}$ depends on the movement direction and speed, and the distance between the UE and the eNB. The larger the value of $T_{R}$, the better quality of service the UE gets when it is handed over to the eNB. The ping-pong effect can also be avoided.

$$
\begin{aligned}
T_{R}=\frac{|A B|}{v} & =\frac{2 R \cos \delta}{v} \\
= & \frac{2 R \sqrt{1-\frac{\left(\left|\overrightarrow{P_{t-1} P_{e}}\right| \times \sin \beta\right)^{2}}{R^{2}}}}{v} \\
= & \frac{2 R \sqrt{R^{2}-\left(\left|\overrightarrow{P_{t-1} P_{e}}\right| \times \sin \beta\right)^{2}}}{v}
\end{aligned}
$$

The strength of the received signal from the eNB is defined as reference signal received power (RSRP), which is the average power of the cell specific reference signal over the entire bandwidth. The received SINR is adopted as the measure of the interference of the UE. The SINR with $i$-th position carrier in the serving cell $s$, $\operatorname{SINR}_{i, s}$, is given by Eq. (3).

$$
S I N R_{i, s}=\frac{R S R P_{R X, i, s}}{I_{i, s}+N_{0} \Delta f}
$$

where $P S R P_{R X, i, s}$ is the received power considering path loss, penetration loss, and shadowing. $I_{i, s}$ is the interference from other cells adopting the same carrier as the serving cell $\_, N_{0}$ is the white noise spectral density, and $\Delta f$ is the bandwidth.

Ranking candidate eNBs using TOPSIS is handled in matrix format. A decision matrix, $D$, is defined as Eq. (4) in which element $x_{i j}$ indicates the performance attribute of alternative $A_{i}$ with respect to decision criterion $C_{j}$. Here the candidates are the neighbor eNBs of the UE, and the decision criteria are $\beta, T_{R}, S I N R$ and $R S R P$. The weights of the decision criteria (denoted as $\left.w_{j}, j=1, \ldots, 4\right)$ are assigned by the decision maker, where $\sum w_{j}=1$. The criterion of high weight means that it has a significant impact in ranking the alternatives. $W$ $=[0.2,0.2,0.3,0.3]$ is used, considering SINR has the same importance as $R S R P$ in deciding the target cell for handover. The procedure of decision making is as follows.

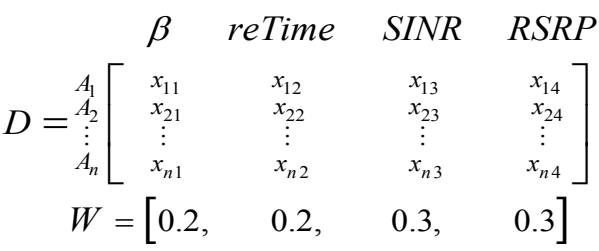

Step 1. Normalize the decision matrix.

The normalization process converts the units into dimensionless units for each decision attribute, $x_{i j}$, using Eq. (5). 


$$
r_{i j}=x_{i j} / \sqrt{\sum_{i=1}^{n} x_{i j}^{2}}, i=1, \ldots, n ; j=1, \ldots, 4
$$

Step 2. Construct the weighted normalized decision matrix.

The weighted normalized value of each element of the normalized decision matrix is obtained with the associated weights by Eq. (6).

$$
v_{i j}=w_{j} r_{i j}, i=1, \ldots, n ; j=1, \ldots, 4
$$

Step 3. Determine the positive-ideal $\left(S^{+}\right)$and negativeideal $\left(S^{-}\right)$solution.

$$
\begin{aligned}
S^{+} & =\left\{v_{1}^{+}, v_{2}^{+}, v_{3}^{+}, v_{4}^{+}\right\} \\
& =\left\{\left(\max _{1 \leq i \leq n} v_{i j} \mid j \in J\right) ;\left(\min _{1 \leq i \leq n} v_{i j} \mid j \in J^{\prime}\right)\right\} \\
S^{-} & =\left\{v_{1}^{-}, v_{2}^{-}, v_{3}^{-}, v_{4}^{-}\right\} \\
& =\left\{\left(\max _{1 \leq i \leq n} v_{i j} \mid j \in J^{\prime}\right) ;\left(\min _{1 \leq i \leq n} v_{i j} \mid j \in J\right)\right\}
\end{aligned}
$$

where $J$ is the set of the benefit criteria (the larger the better) and $J$ is the set of the cost criteria (the smaller the better). Here, $J=\left\{T_{R}, \operatorname{SINR}, R S R P\right\}$ and $J^{\prime}=\{\beta\}$.

Step 4. Calculate the distance of each alternative from $S^{+}$and $S$.

$$
\begin{aligned}
& d_{i}^{+}=\sqrt{\sum_{j=1}^{4}\left(v_{i j}-v_{j}^{+}\right)^{2}}, i=1, \ldots, n \\
& d_{i}^{-}=\sqrt{\sum_{j=1}^{4}\left(v_{i j}-v_{j}^{-}\right)^{2}}, i=1, \ldots, n
\end{aligned}
$$

Step 5. Measure the relative closeness to $S^{+}$.

$$
C_{i}^{+}=\frac{d_{i}^{-}}{d_{i}^{+}+d_{i}^{-}}, i=1, \ldots, n
$$

Step 6. Rank the alternative eNBs by $C_{i}^{+}$.

The candidate cells are ranked in a descending order of $C_{i}^{+}$. The best one for handover has the shortest distance to the positive-ideal solution.

\subsection{Handover Time}

Unlike symmetric macro-only network, the deployment of pico cells inside a macro cell causes the network structure to become asymmetric. Moving from one cell to another in homogeneous network, the received signal power of a UE from the serving eNB decreases while that from the target eNB increases. However, in HetNet environment, there exist four different scenarios of the movement of a UE as shown in Fig. 3.

(1). Moves away from macro cell (MCell) towards pico cell (PCell), and thus the received power from its serving macro eNB (MeNB) decreases while that from the target pico eNB (PeNB) increases

(2). Moves into PCell from the cell edge of MCell, and thus increasing signal from both of them

(3). Moves away from PCell towards MCell, and thus increasing signal from $\mathrm{MeNB}$ and decreasing signal from the serving PeNB

(4). Moves away from both MeNB and PeNB, and thus decreasing signal from both of them. Note that it is inappropriate to employ the same handover decision mechanism of homogeneous network to the HetNet due to the asymmetric nature.

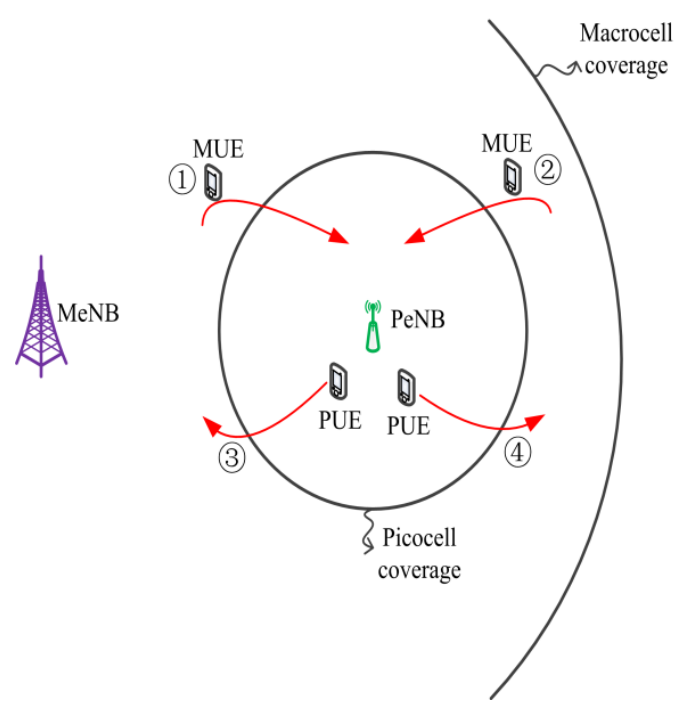

Fig. 3. Four different scenarios of UE movement.

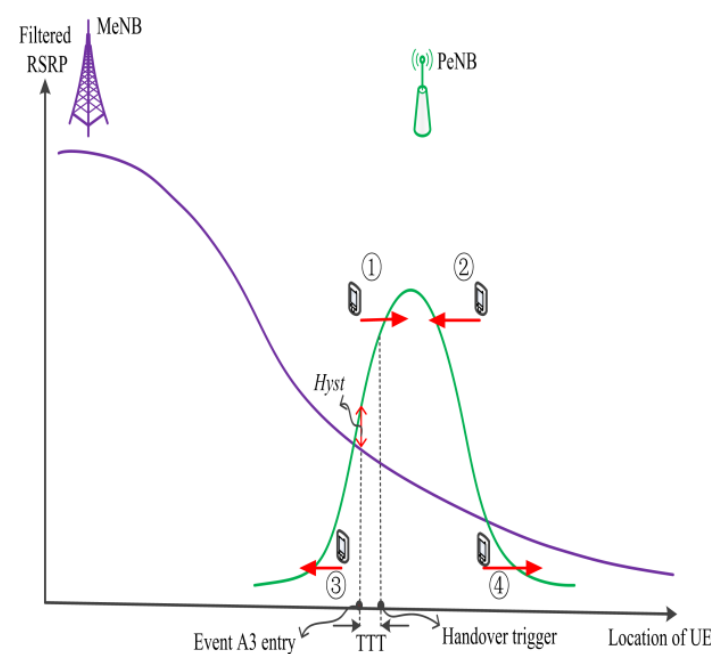

Fig. 4. Handover in HetNet for the four cases of Fig. 3.

Deploying PeNBs inside an MCell is to offload the services in hot spot or cell edge and thus relieve the burden of the cell. It improves the capacity of the 
network and quality of service of the UE. However, dense PeNBs could detriment the SINR of the UE. As shown in Fig. 4, for the UE of case (1) and (2), the signal from the target PeNB deteriorates the SINR of the UE. The UE will encounter RLF if its SINR is smaller than the threshold. A UE is considered to be out of synchronization when its wideband SINR falls below $Q_{\text {out }}(-8 \mathrm{~dB})$ and to be back in synchronization when it reaches $Q_{\text {in }}(-6 \mathrm{~dB})$ [8].

The four cases above are categorized into two classes, in-pico and out-pico handover. When a UE moves into PCell (1), even though it receives a reasonably strong signal from its serving MeNB, the signal from PeNB can be a strong interference, degrading the quality of service and even causing RLF for the UE. As shown in Fig. 5, handover is made even before TTT expires when the downlink SINR of the UE becomes very small as expressed in Eq. (10).

$$
\operatorname{SINR}<\operatorname{SINR}_{R L F}
$$

where $\operatorname{SINR}_{R L F}$ is a threshold value. An RLF event will be declared when the downlink SINR of the UE has been out of synchronization and stayed below $Q_{\text {in }}$ for the duration of the T310 time window [8]. $\operatorname{SINR}_{R L F}$ is set to $-6 \mathrm{~dB}$ in the proposed scheme.

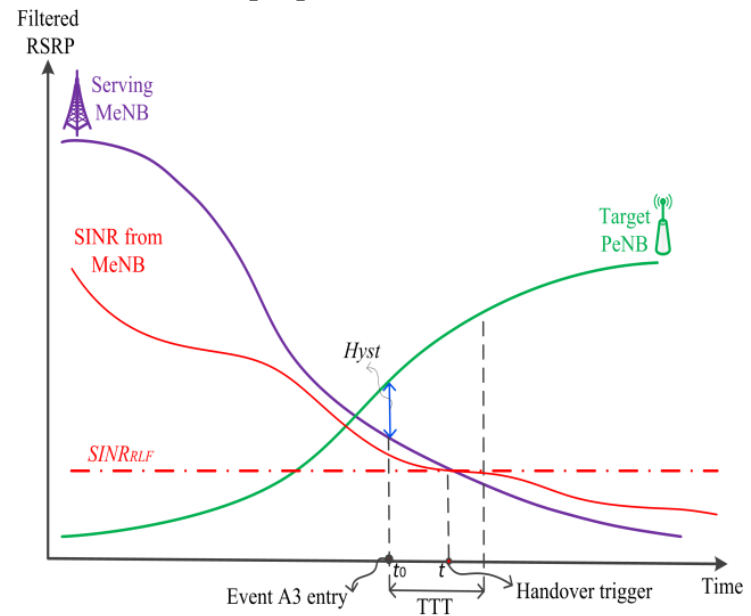

Fig. 5. The handover mechanism for in-pico scenario.

When a UE moves out from PCell towards MeNB, as the case (3) in Fig. 3, the signal power from MeNB increases rapidly while that from the serving PeNB deteriorates badly because of its limited transmission power. It is necessary to make a handover early for the UE to avoid RLF caused by the connection to the source PeNB of poor quality. Handover is thus triggered in the proposed scheme even before the TTT window expires if the rate of the change of the difference in RSRP is higher than a threshold. This is to let the real-time applications avoid RLF caused by excessively low signal power. Fig. 6 shows the relation between the factors used in the proposed scheme for this handover scenario.

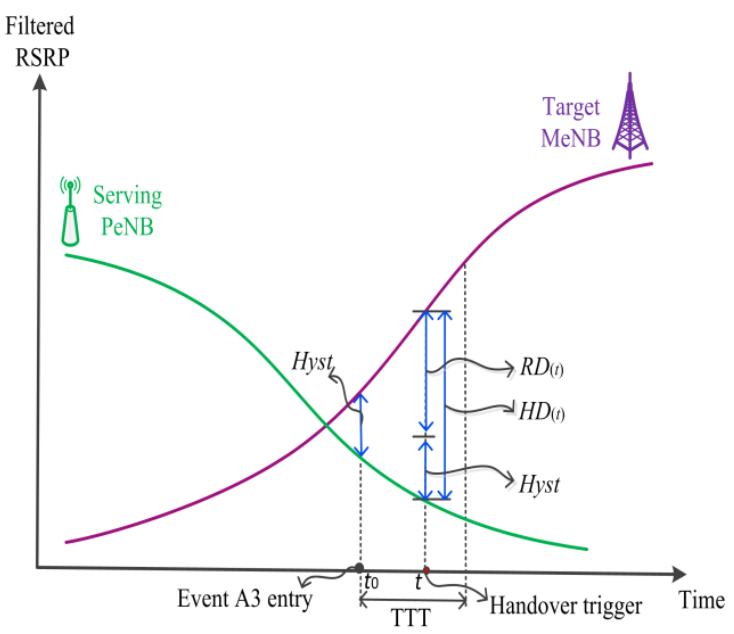

Fig. 6. The handover mechanism for out-pico scenario.

As shown in Fig. 6, $R D(t)$ indicates the difference in the RSRPs of the UE between the target eNB, $M$, and serving eNB, $P$, calculated by Eq. (11). $H D(t)$ is defined as the difference between $R D(t)$ and Hyst when the UE gets service from the serving $\mathrm{PeNB}_{-} P$ at time $t$, calculated by Eq. (12).

$$
\begin{aligned}
& R D(t)=R S R P_{M}(t)-R S R P_{P}(t) \\
& H D(t)=R D(t)-H y s t
\end{aligned}
$$

Filtered $R D(t), F R D(t)$, is obtained by Eq. (13) using the first order auto-regressive (FOAR) moving model during each measurement period. Similarly, filtered $H D(t), F H D(t)$, is obtained by Eq. (14). The use of FOAR allows high channel quality by considering the signal strengths in the previous handover measurement periods. The rate of increase of $F H D(t), R(t)$, is determined from Eq. (15). The relative importance of the current and prior measurement period in deciding $F R D(t)$ and $F H D(t)$ is controlled by the averaging factor, $\beta$. Here $\beta$ is chosen depending on the measurement period $\left(T_{m}\right)$ and TTT, as $\beta=T_{m} / T T T$. A mathematical analysis in [14] shows choosing $\beta$ in the differential integration by the FOAR model in the proposed scheme provides a stationary stochastic process of $F R D(t)$ and $F H D(t)$. 


$$
\begin{gathered}
F R D(t)=(1-\beta) F R D(t-1)+\beta R D(t) \\
F H D(t)=(1-\beta) F H D(t-1)+\beta H D(t) \\
R(t)=\frac{F H D(t)-F H D(t-1)}{F H D(t-1)}
\end{gathered}
$$

Handover is triggered for out-pico scenario even before the TTT window is over when Eq. (16) is satisfied.

$$
\left\{\begin{array}{l}
F H D(t)>\beta H y s t \\
R(t)>\beta
\end{array}\right.
$$

For the handover mechanism not employing the TTT window, if a UE handed over to another eNodeB returns to the host shortly, the signaling resources are wasted substantially due to frequent channel connection operations. In addition, packet loss and delay are increased due to data forwarding. On the other hand, handover based on TTT can decrease the system throughput since the handover occurs 1) to the target PeNB from the serving MeNB incurring RLF due to dramatically degraded SINR of the UE; or 2) to the target MeNB of better quality from the serving PeNB of poor quality. The proposed scheme effectively resolves these issues using new factors and decision mechanism.

In summary, handover is triggered for the UE to the best candidate cell ranked by TOPSIS procedure if the conditions listed in Table 1 are satisfied. Note that, for both in-pico and out-pico scenario, if handover is not triggered by Condition 1 or 2, Condition 3 (as the basic scheme) is used to decide when to trigger a handover using the filtered value of the signal, $F R D$.

Table 1. The rules used to decide handover.

\begin{tabular}{|c|c|c|}
\hline & TTT window & Signal power \\
\hline Scenario 1 & $T T T_{\text {_timer }}<T T T$ & $S I N R(t)<$ SINR $R_{R L F}$ \\
\hline Scenario 2 & $T T T_{\text {_timer }}<T T T$ & $F H D(t)>\beta H y s t \&$ \\
& & $R(t)>\beta$ \\
\hline Scenario 3 & $T T T_{-}$timer $\geq T T T$ & $F R D(t)>H y s t$ \\
\hline
\end{tabular}

\section{Performance Evaluation}

In this section the proposed scheme is validated by computer simulation, and compared with the standard hard scheme and the network control handover $(\mathrm{NCH})$ scheme [15]. The NCH scheme also adopts SINR threshold in handover decision for both in-pico and outpico scenario. The simulations are carried out on a Linux machine with a $2.9 \mathrm{GHz} \mathrm{CPU}$ and $4 \mathrm{~GB}$ of RAM using an open-source system level simulator, LTE-Sim [16].

\subsection{Simulation Environment}

The parameters used in the simulation are listed in Table 2, which are mainly based on the 3GPP Study Item "HetNet mobility enhancements for LTE" [3]. A rural macro network of 19 hexagonal cells of the radius of $0.5 \mathrm{~km}$ with one pico cell of the radius of $0.1 \mathrm{~km}$ deployed on each border is used as the target network of the simulation. Refer to Fig. 8. The total transmission power of MeNB is $46 \mathrm{dBm}$ and that of PeNB is $30 \mathrm{dBm}$. The channel model is implemented with path loss, shadow fading, penetration loss and fast fading.

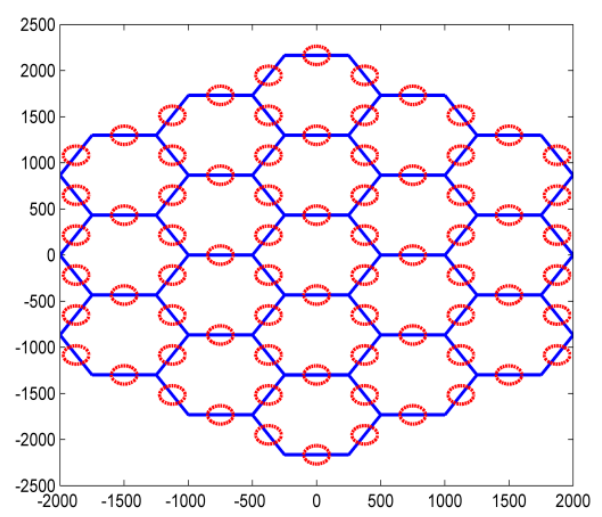

Fig. 8. The network employed for the simulation.

Table 2. The parameters of the network.

\begin{tabular}{|c|c|}
\hline Parameter & Value \\
\hline Cellular layout & $\begin{array}{c}\text { MCell: Hexagonal grid of 19 cells } \\
\text { PCell: 72 cells, each one fixed } \\
\text { on the border of the MCells }\end{array}$ \\
\hline Radius & $\begin{array}{c}\text { MCell: } 500 \text { meters PCell: } 100 \\
\text { meters }\end{array}$ \\
\hline Carrier frequency & $2.0 \mathrm{GHz}$ \\
\hline Bandwidth & $10 \mathrm{MHz}$ \\
\hline RB width & $180 \mathrm{KHz}$ \\
\hline eNB TX power & $\begin{array}{c}\text { MCell: } 46 \mathrm{dBm} \\
\text { PCell: } 30 \mathrm{dBm}\end{array}$ \\
\hline Path loss model & $\begin{array}{c}\text { MCell: } 128.1+37.6 \log _{10}(d) \\
\text { PCell: } 140.7+36.7 \log _{10}(d) \\
d \text { is in kilometers }\end{array}$ \\
\hline Penetration loss & MCell: $20 \mathrm{~dB}$ \\
\hline Phadowing fading & MCell: $8 \mathrm{~dB} \mathrm{~dB}$ \\
\hline RSRP period & $10 \mathrm{~ms}$ \\
\hline BS noise figure $10 \mathrm{~dB}$ \\
\hline UE noise figure & $5 \mathrm{~dB}$ \\
\hline Duplexing & $9 \mathrm{~dB}$ \\
\hline Simulation time & FDD \\
\hline SINR & $75,000 \mathrm{~ms}$ \\
\hline
\end{tabular}

The parameters of the traffic are listed in Table 3. There are initially $\{1,5\}$ UEs uniformly distributed in each MCell (total $\{19,95\}$ UEs in the area). They move at a constant speed in random direction. The mobility 
model of random direction may cause the UEs to travel to the edge of the simulation area before changing the direction and speed, which realistically model the UE in an open area. Each UE holds a VoIP flow for 70,000 ms during the entire simulation time of $75,000 \mathrm{~ms}$. The time gap guarantees that there is enough time for the packet sent last to arrive at the destination. However, a packet is regarded as invalid if it is received later than $100 \mathrm{~ms}$ after transmission. The speed of UE is assumed to be constant $\{3,30,120,200,300 \mathrm{kmph}\}$ during the entire simulation, modeling normal pedestrian ( $3 \mathrm{kmph})$, car (30 kmph), normal-speed train (120 kmph), highspeed rail (200 kmph and even $300 \mathrm{kmph}$ such as Korea Train Express (KTX) [17]), respectively. Hyst of $2 \mathrm{~dB}$ and TTT of $160 \mathrm{~ms}$ are used as [3].

Table 3 . The parameters of the traffic.

\begin{tabular}{|c|c|}
\hline Parameter & Value \\
\hline Number of UEs & $\{1,5\}$ UEs per MCell initially \\
\hline UE speed & $\{3,30,120,200,300\}$ kmph \\
\hline UE mobility & Random direction \\
\hline Data traffic & 1 VoIP flow per UE \\
\hline $\begin{array}{c}\text { Duration of } \\
\text { VoIP flow }\end{array}$ & $70,000 \mathrm{~ms}$ \\
\hline Maximum delay & $100 \mathrm{~ms}$ \\
\hline $\begin{array}{c}\text { Handover } \\
\text { hysteresis }\end{array}$ & $2 \mathrm{~dB}$ \\
\hline TTT & $160 \mathrm{~ms}$ \\
\hline
\end{tabular}

The handover performance is measured by average number of handover per second $(H O F)$, handover frequency for specific type of handover, packet loss rate $(P L R)$, and goodput per UE $(G D P)$. They are defined as follows.

$$
H O F=\frac{N}{T}
$$

where $N$ is the total number of handovers and $T$ is the simulation time.

$$
P L R=\left(1-\frac{\text { pack }_{\text {rec }}-\text { pack }_{\text {inv }}}{\text { pack }_{\text {sent }}}\right) \times 100 \%
$$

where pack $_{\text {sent }}$ is the number of packets generated by the sender eNB, pack $k_{\text {rec }}$ is the number of packets which finally arrive at the destination, and packinv is the number of packets arriving at the destination beyond the delay bound of $100 \mathrm{~ms}$.

$$
G D P=\frac{\sum_{j=1}^{J} g d p_{j}}{J}
$$

where $g d p_{j}$ is the total size of packets (in bit) correctly received by $\mathrm{UE} j$ during the simulation time, and $J$ is the total number of UEs.

\subsection{Simulation Results}

Figure 7 shows the number of handovers per second for the three schemes with 19 UEs and 95 UEs, respectively. It is obvious that the more UEs in the network, the more handover occurs. The $\mathrm{NCH}$ scheme demonstrates the largest number of handovers for both the scenarios of 19 and 95 UEs. This is because the handover decision based on only the SINR threshold causes frequent handover especially in the HetNet environment where the UE of poor signal power can find several neighbor eNBs of high signal power. The basic hard scheme can somewhat limit the number of handovers by triggering handover only when the received signal is larger than handover hysteresis for the entire duration of TTT window. Observe that the proposed scheme shows minimum number of handover among the three. It can also be seen from Fig. 7 that the number of handovers increases along with the speed of the UE, especially for the NCH scheme. In the eNBredundant HetNet environment, UE can easily reach the condition where the received signal from its serving eNB is strong enough for stable communication but severely interfered from the neighbors having strong signal.

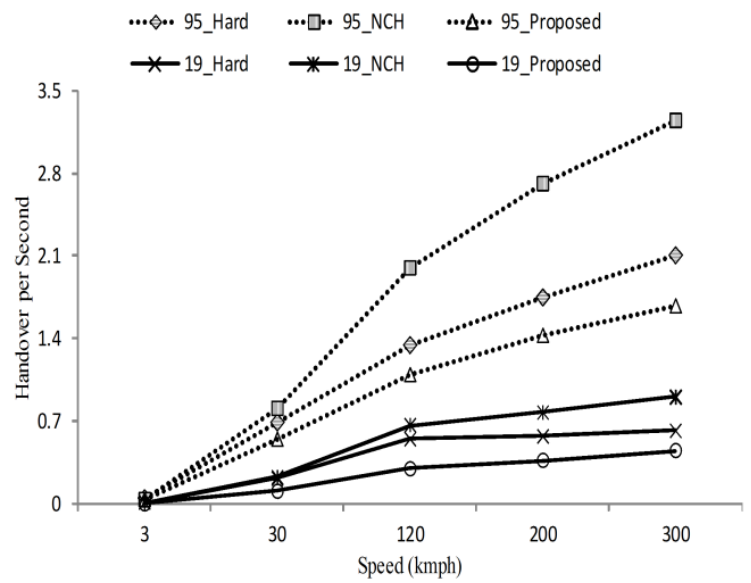

Fig. 7. The comparison of the number of handovers per second.

The number of handovers of three different types of handovers in HetNet environment with 95 UEs is presented in Fig. 8. The three types are macro-to-macro (M2M), macro-to-pico (M2P), pico-to-macro (P2M). There is no pico-to-pico $(\mathrm{P} 2 \mathrm{P})$ handover since there is no overlap of coverage of PCells in the simulated network where the PCells of radius of 100 meters are deployed at the border of the MCells of radius of 500 
meters. For the basic handover scheme, the handover frequency of M2M is always the largest and it increases as the UE speed grows. When a UE moves form one MCell to another through a PCell deployed on the boundary, the UE of high speed just passes through the PCell before the TTT window expires, decreasing M2P and $\mathrm{P} 2 \mathrm{M}$ handover. For the $\mathrm{NCH}$ scheme, the handover frequency of M2P and P2M dramatically increases as the speed of the UE increases. Recall that the downlink SINR of the UE using the NCH scheme falls below the threshold when it moves across different types of eNBs. On the contrary, even though the handover frequency of $\mathrm{M} 2 \mathrm{P}$ and $\mathrm{P} 2 \mathrm{M}$ with the proposed scheme increases as the UE speed increases, it is significantly less than that of the $\mathrm{NCH}$ scheme due to the prediction of the target. The proposed scheme mitigates the cases where the UE moves into a neighbor cell of similar signal power as its serving cell but moves out soon. Also note that the total number of handovers is less than that of the other schemes.

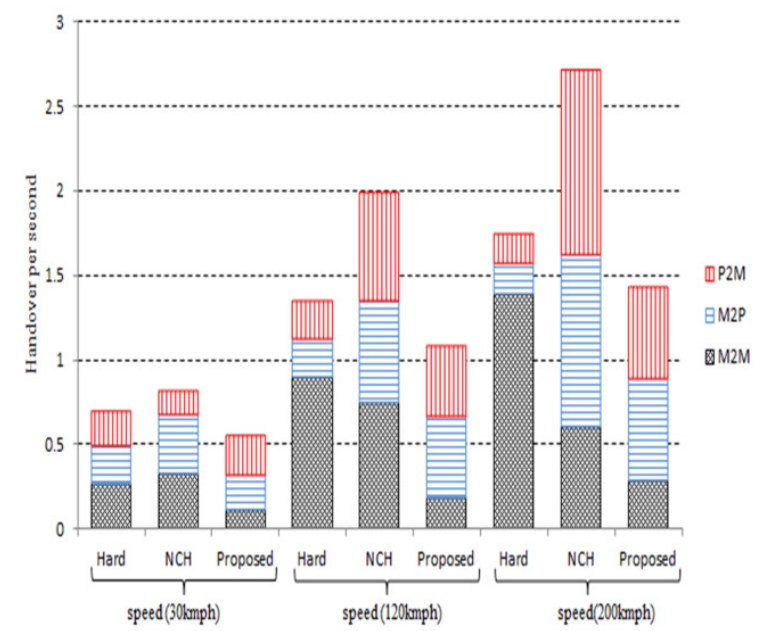

Fig. 8. The handover frequency with different scenarios.

Figure 9 compares the packet loss rate with different speeds and numbers of UEs. The packet loss rate for all the schemes increases with the increase of the speed of the UE, while the proposed scheme shows much less packet loss rate than the others. Note that for both the cases of 19 and 95 UEs, the standard scheme shows the worst result when the speed gets high. This is because the UEs get out of the radio coverage of the serving eNB before it is handed over to the target one, especially for the ones of high speed. The $\mathrm{NCH}$ scheme can deal with the case where the UE encounters degraded SINR. However, since the PeNBs locate at the border of the coverage of MeNB, the received power of the signal from its serving MeNB is very low when the UE moves into the PCell. Even in this case, the proposed scheme can effectively make handover decision, triggering handover when the power of the signal received from the target PCell and the increasing rate of the power are high enough. The proposed scheme also shows good performance in handling a large number of UEs. The increase of packet loss rate with the proposed scheme when the number of UEs grows to 95 from 19 is the smallest among the three schemes. The proposed scheme predicts the target eNB along with the UE movement and makes early handover to a suitable target eNB when the received power or its downlink SINR is very small, avoiding RLF and reducing packet loss rate.

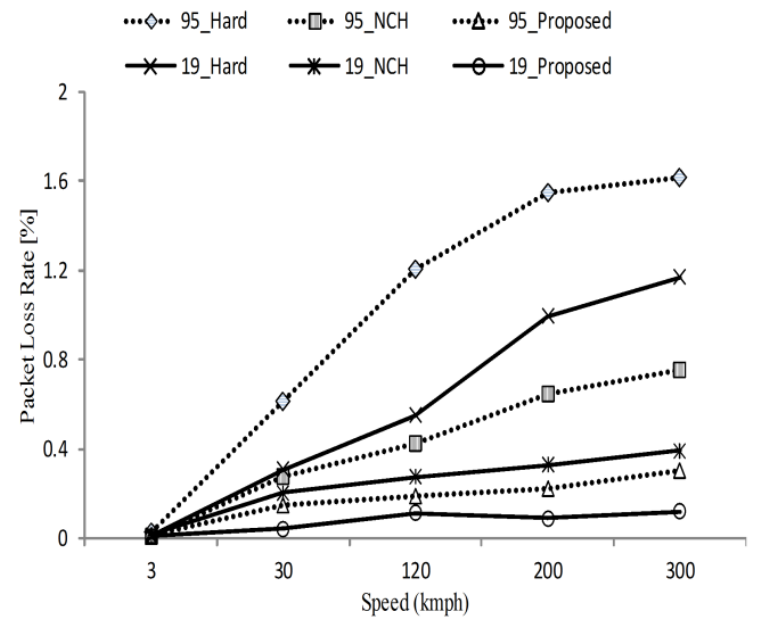

Fig. 9. The comparison of packet loss rates.

Figure 10 shows the comparison of average goodput per UE for the schemes with different speeds. As expected, the proposed scheme gains the best goodput since it triggers prompt handover to select the cell of good quality where the UE will eventually stays for long enough. The handover mechanism triggering handover whenever the downlink SINR is less than a threshold may frequently incur unnecessary handover. Frequent switching from one cell to another wastes the resource and interrupts the service of the UE with the $\mathrm{NCH}$ scheme. The goodput of the UE using the standard scheme decreases dramatically, especially when the speed is high. This is because the UE experiences a high probability of RLF due to either too poor signal strength or degraded SINR interfered by neighbor eNBs. 


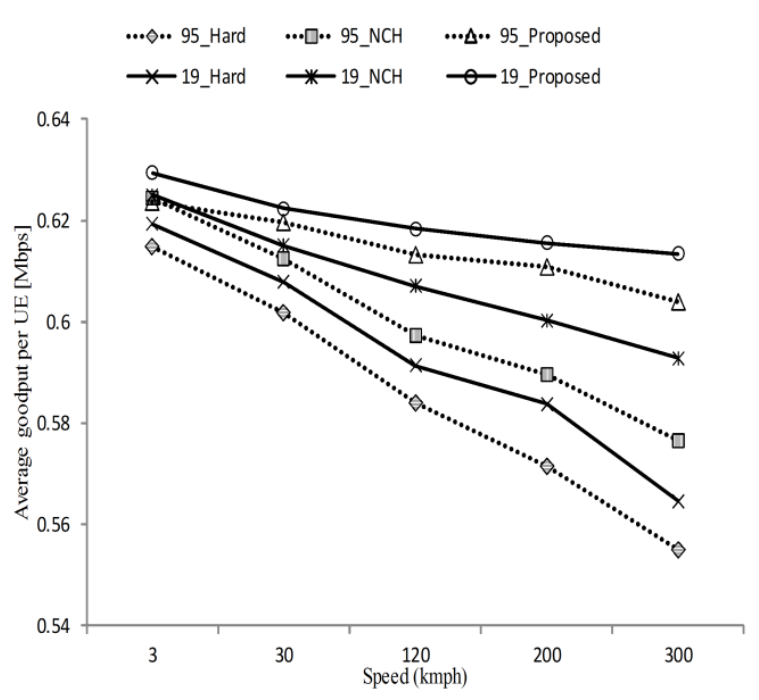

Fig. 10. The comparison of goodputs per UE.

\section{Conclusion and Future Work}

In eNB-rich HetNet environment the signal power of the neighbor eNBs plays an important role in making handover decision. The proposed scheme triggers handover for the UE any time even before the TTT window is over, which receives relatively strong signal from its serving eNB but is badly interfered by the neighbors to avoid RLF caused by deteriorated SINR. The proposed scheme also triggers handover promptly when the received signal power is too low while a suitable target eNB of good quality is decided. The best candidate cell for handover is decided by an MCDM algorithm based on the movement direction, residence time, downlink SINR, and received RSRP of the UE. The simulation results reveal that the proposed scheme shows higher goodput, lower packet loss rate, and reduced handover than the existing schemes.

The network topology with pico cells deployed at the border of the macro cells was studied in this paper. Note that the cells of small power can also be located at crowded region inside the coverage of macro cell. As future work, the performance of the proposed scheme is further enhanced with the network layout where pico cells are randomly distributed in the macro cells, too. The number of pico cells per macro cell displaying the best result will also be investigated to extend the proposed scheme.

\section{Acknowledgment}

This research was supported by Basic Science Research Program through the National Research Foundation of Korea (NRF) funded by the Ministry of Education, Science and Technology (2012R1A1A2040257 and 2013R1A1A2060398), the second Brain Korea 21 PLUS project, ICT R\&D program of MSIP/IITP (1391105003), and Samsung Electronics (S-2014-0700000). Corresponding author: Hee Yong Youn.

\section{References}

[1] Cisco VNI, "Cisco Visual Networking Index: Global Mobile Data Traffic Forecast Update, 2014-2019," Cisco Public Information, Feb. 2015.

[2] X. Chen et al, "Efficient and Prompt Handover in LTEBased Systems by Predicting the Target eNodeBs," IEEE Int'l. Cyber-Enabled Distributed Computing and Knowledge Discovery (CyberC), pp. 406-413, Oct. 2014.

[3] 3GPP, "Mobility Enhancements Heterogeneous Networks," 3GPP TR 36.839 (v11.1.0), Dec. 2012.

[4] K. Kitagawa et al, "Performance evaluation of handover in LTE-Advanced systems with pico cell Range Expansion," IEEE Int'l Symp. on Personal Indoor and Mobile Radio Communications (PIMRC), pp. 10711076, Sept. 2012.

[5] D. Lopez-Perez et al, "Mobility Enhancements for Heterogeneous Networks through Interference Coordination," 2012 IEEE WCNCW on Broadband Femtocell Techn., pp. 69-74, Apr. 2012.

[6] J. Turkka et al, "Self-optimization of LTE Mobility State Estimation thresholds," 2014 IEEE WCNCW on Self-Organizing Networks, pp. 161-165, Apr. 2014.

[7] S. Barbera et al, "Improved mobility performance in LTE co-channel HetNets through speed differentiated enhancements," IEEE Globecom Workshop, pp. 426430, Dec. 2012.

[8] D. Lopez-Perez et al, "Mobility management challenges in 3GPP heterogeneous networks." IEEE Commun. Mag., 50(12), pp. 70-78, Dec. 2012.

[9] M. Peng et al, "Self-configuration and self-optimization in LTE-advanced heterogeneous networks," IEEE Comm. Mag., 51(5), pp. 36-45, May 2013.

[10] L. Ewe and H. Bakker, "Base station distributed handover optimization in LTE self-organizing networks," IEEE Int'l Symp. on Personal Indoor and Mobile Radio Communications (PIMRC), pp. 243-247, Sept. 2011. 
[11] K. Kitagawa et al, "A handover optimization algorithm with mobility robustness for LTE systems," IEEE Int'l Symp. on Personal Indoor and Mobile Radio Communications (PIMRC), pp. 1647-1651, Sept. 2011.

[12] C. Ma et al, "Inter-Tier Handover in Macrocell-RelayFemtocell Heterogeneous Networks," IEEE 75th Vehicular Technology Conference (VTC Spring), pp. 15, May 2012.

[13] X. Chen et al, "DIHAT: Differential Integrator Handover Algorithm with TTT window for LTE-based systems," EURASIP JWCN, 2014.1: 1-13, Oct. 2014.

[14] L. Anojkumar et al, "Comparative analysis of MCDM methods for pipe material selection in sugar industry," Expert Systems with Applications, 41(6), pp. 2964-2980, 2014.

[15] G. Zhou et al, "A network controlled handover mechanism and its optimization in LTE heterogeneous networks," IEEE WCNC, pp. 1915-1919, Apr. 2013.

[16] G. Piro et al, "Simulating LTE Cellular Systems: An Open-Source Framework," IEEE Trans. Veh. Tech, 60(2): 498-513, Feb. 2011.

[17] Korea Train Express (KTX). http://info.korail.com/ mbs/english/subview.jsp?id=english_050101000000. 